\title{
The impact of nasal surgery on air-conditioning of the nasal airways*
}

\author{
Konrad G. Kastl ${ }^{1}$, Gerhard Rettinger ${ }^{1}$, Tilman Keck $^{2}$ \\ Department of Otorhinolaryngology, University of Ulm, Ulm, Germany \\ 2 Department of Otorhinolaryngology, Head, Neck, and Facial Plastic Surgery, Elisabethinen Hospital GmbH, \\ Academic Hospital of the Medical University of Graz, Graz, Austria
}

\begin{abstract}
SUMMARY
Air-conditioning in the nasal passageways is one of the most important functions of the upper airways. By means of in-vivo-measurements and numerical simulation, the air-conditioning function of the nose has been extensively investigated. Less well known is the effect of nasal surgery on the nasal climate. The following study presents a summary of the effect of various rhino-surgical operations, i.e. turbinoplasty, septoplasty, septorhinoplasty, repair of septal perforations, functional and radical sinus surgery, on the air-conditioning function of the nose. Nasal and sinonasal interventions have been demonstrated to be associated with increased nasal heating and humidification when the mucosal lining is preserved. Radical interventions with reduction of turbinate tissue cause reduced nasal warming and moistening within the nasal airway, with increased risk of nasal dryness and crusting. Although the impact of the nasal cycle and the airflow distribution within the nasal cavity on nasal temperature and humidity distribution is not fully understood yet, too much widening of the nasal cavity by sinunasal interventions has carefully to be avoided.
\end{abstract}

Key words: nasal air-conditioning, nasal surgery, nasal climate, empty nose syndrome

\section{INTRODUCTION}

Nasal air-conditioning is one of the most important functions of the nose. During inspiration the intranasal air is heated, humidified, and cleaned from larger particles ${ }^{(1,2) .}$ This way, the nose plays an important role in the protection of the lower airways ${ }^{(3)}$. In case of restricted nasal breathing or during increased physical effort, breathing trough the mouth is necessary. Considerable disadvantages result when the nasal airway is excluded form respiration. Svensson et al. show that the extent of water loss with expiration through the mouth, was $42 \%$ higher than with nose-breathing ${ }^{(4)}$. This way reduced nasal air-conditioning may result in disease of the lower airways. Subjects with asthma show a correlation to nasal functional disturbances ${ }^{(5)}$. Impaired nasal breathing is subjectively very uncomfortable and compromises the quality of life. Depending on the pathology, surgery to improve nasal breathing should result in a subjective and objective benefit for the patient. There are various studies presenting an overview of the capacity and mechanisms of nasal air-conditioning ${ }^{(1,3,6,7)}$. The basic mechanisms of nasal conditioning and effects of nasal pathologies on nasal conditioning have been intensively investigated. However, the influence of rhino-surgery is widely neglected ${ }^{(1,3,6,7)}$.

This review reports the current knowledge of the effects of various rhino-surgical methods with regard to their effects on nasal air-conditioning.

\section{TURBINATE SURGERY}

The nasal turbinates play an important role in intranasal climatization ${ }^{(7-9)}$. Obstructed nasal breathing is often targeted by surgical intervention on the turbinates.

Many studies have been conducted on the effects of turbinate surgery to improve nasal breathing. The results are, in some cases, difficult to compare as a great number of different operating techniques are described ${ }^{(10-13)}$. At the same time most studies are based on questionaires ${ }^{(14)}$ or function tests like active anterior rhinomanometry or acoustic rhinometry ${ }^{(15)}$. Parameters of nasal air-conditioning, such as temperature or humidity are not recorded with these techniques.

Another aspect is the fact that turbinate surgery is often carried out in combination with other rhino-surgical techniques like septoplasty, septorhinoplasty or sinus surgery. In this case it is often not possible to determine how the result can be credited to turbinate surgery or to other surgical procedures. Naftali et al. described the proportion of influence of anatomic structures on the climatic performance of the nose in various computer simulations ${ }^{(7)}$. The influence of the inferior and middle turbinates is estimated to be $15 \%$ and that of the nasal septum to be $25 \%$. In the computer simulation, the removal of the middle turbinate lead to a reduction of $12 \%$ in nasal air conditioning efficiency. In case of removal of the inferior turbinate, the result was $16 \%$ lower. It can be seen, that the results with nose-like models remain relatively inexact as anatomical struc- 


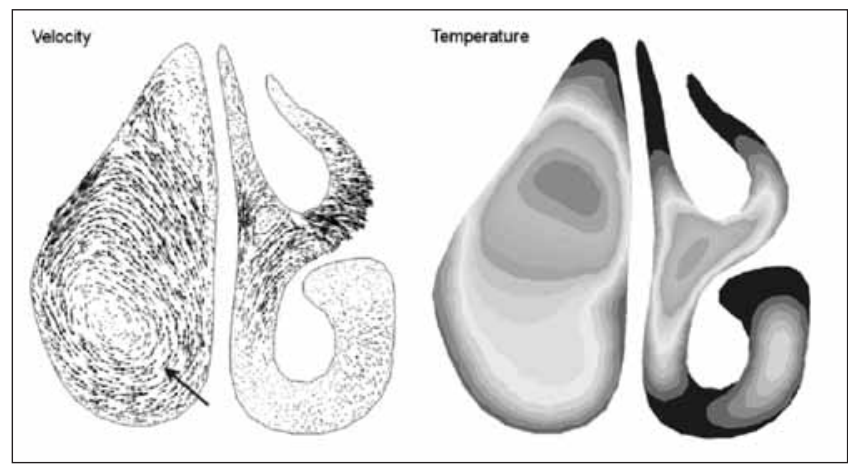

Figure 1. Numerical simulation of velocity (left) and temperature (right) after unilateral resection of the turbinates during inspiration. A spacious vortex (arrow) and a disturbed temperature distribution within the "operated" right nasal cavity demonstrated ${ }^{(16)}$.

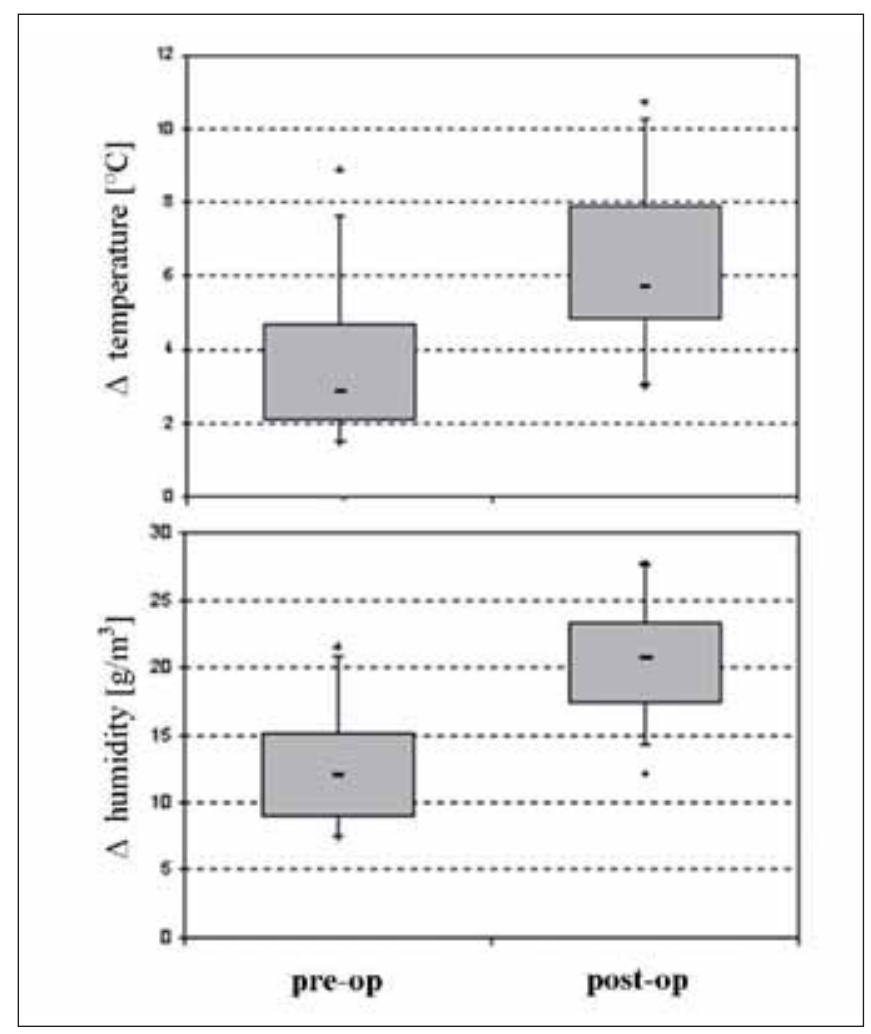

Figure 2. Box-Whisker-plot showing the temperature and humidity increase before and after septoplasty measured in the anterior turbinate area ${ }^{(22)}$.

tures of the nose are only approximately simulated by simple geometric shapes.

In another study, the effect of turbinate resection on intranasal temperature and airflow is simulated in an anatomical computer model with noticeably higher accuracy ${ }^{(16)}$. Based on CT scan data of healthy subjects, one side of the nose remained untouched, on the other side the inferior and middle turbinate were removed to simulate the effects of surgical resection. On the side of the turbinate resection a large vortex developed (Figure 1). As a result, in comparison to the non-modified side, the temperature exchange between the air within the nasal airways and mucosal lining of the nose significantly decreased. This model very well describes the so called "empty nose" and is comparable to the situation after radical sinus surgery.

In a study from 1942, Moe examines the effect of nasal interventions to enlarge the nasal cavities, mainly with turbinate resections, on the respiratory function of the nose ${ }^{(17)}$. He could not find a significant decrease of temperature and humidity in the nasopharynx compared to controls. However, the operated noses showed a noticeably lower transnasal increase in temperature and humidity. The extent of the functional reduction appears to be determined by the widening of the nasal cavities ${ }^{(17)}$. Despite of the fact that these results have to be critically viewed because of the technical possibilities at that time, they underline the importance of the turbinates for the intranasal climatization.

\section{SEPTOPLASTY}

There is sufficient evidence for the subjective improvement of nasal breathing after septoplasty ${ }^{(18,19)}$. Several studies, however, show that this improvement of nasal breathing only poorly correlates with the objectively measured results ${ }^{(20,21)}$. Studies on the effects of septoplasty on the climatization-function of the nose are rare. A recent study showed a statistically significant increase in end-inspiratory temperature and in relative humidity after septoplasty ${ }^{(22)}$. Intranasal temperature and relative humidity were continuously measured during several breathing cycles close to the head of the middle turbinate. It was shown for the first time that septoplasty with bilateral tubinoplasty leads to a significant improvement of the climatization function of the nose (Figure 2). To what extent surgery of turbinates is responsible and to what extent the septum correction is responsible, remains open at this point.

\section{SEPTORHINOPLASTY}

There are several studies on the effects of septorhinoplasty on nasal breathing. In most studies an improvement in nasal breathing after surgery is registered ${ }^{(23)}$. The situation in the literature is comparable to septoplasty. There are only few studies on the effect of septorhinoplasty on air-conditioning. However, conditioning in the nasal passageways after rhinoplastic and not only septal surgery may differ from nasal airconditioning after solely septal surgery because of the extended surgical interventions that are often performed in rhinoplasty, e.g. widening of the nasal valve area by lateral osteotomies or other interventions on the nasal pyramid. In a prospective study, patients were examined before and 4 to 6 months after

Table 1. Summary of the most important results of Rozsasi et al. ${ }^{(24)}$.

\begin{tabular}{lccc}
\hline mean & pre-SRP & post-SRP & p-value \\
\hline Temperature $\left[{ }^{\circ} \mathrm{C}\right]$ & 27.0 & 29.4 & 0.003 \\
Total water content $[\mathrm{mg} / 1]$ & 21.6 & 26.3 & 0.003 \\
Heat increase $\left[{ }^{\circ} \mathrm{C}\right]$ & 2.9 & 6.2 & 0.003 \\
Water gradient $[\mathrm{mg} / 1]$ & 11.9 & 20.6 & 0.001 \\
\hline
\end{tabular}


septorhinoplasty and inferior turbinoplasty with regard to the temperature and the relative humidity increase ${ }^{(24)}$. In comparison to the pre-operative situation, postoperative values showed a significantly higher nasal airflow as measured by active anterior rhinomanometry. The post-operative nasal temperature, the heat increase, the total water content, and the water gradient were significantly higher compared to the pre-operative values (Table 1).

Patients with internal and external nasal deformities showed a noticeable improvement of intranasal air-conditioning after septorhinoplasty. However, despite of convincing results, further studies with larger patient groups without simultaneous turbinate surgery have to be performed.

\section{SEPTAL PERFORATION}

Patients with septal perforations suffer from nasal blockage, crusting, recurrent epistaxis or whistling ${ }^{(25)}$. In particular, nasal blockage is difficult to test as results of active anterior rhinomanometry or acoustic rhinometry in these patients are not reliable ${ }^{(26)}$. In patients with septal perforation a significantly minor increase in humidity during inspiration is found compared to healthy controls ${ }^{(27)}$. Numerical simulations demonstrated the formation of vortexes, causing a disturbed airflow and temperature distribution within the nose ${ }^{(28)}$.

In a prospective study, conditioning measurements were performed immediately prior to and approximately 7 months after surgical closure of septal perforations ${ }^{(29)}$. Post-operatively, intranasal temperature and humidity values increased significantly (Figure 3). At the same time, a significant reduction of epistaxis and nasal dryness as registered by a symptom score could be observed. A successful surgical closure of septal per-

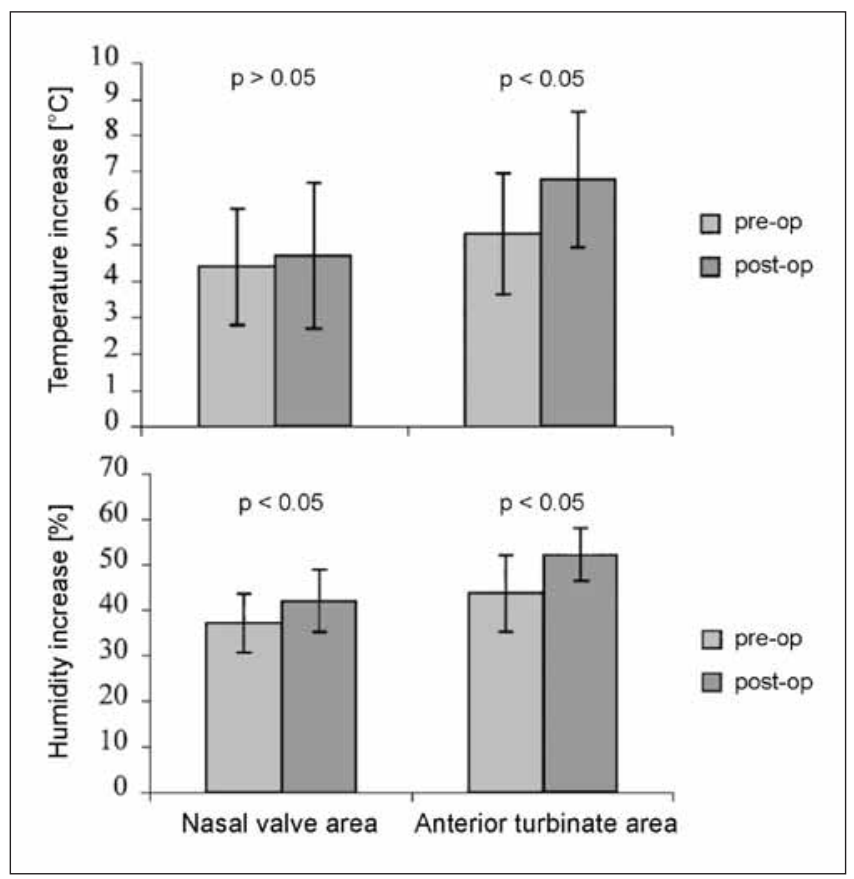

Figure 3. Transnasal temperature and humidity increase in patients with nasal septal perforation before and after surgical closure ${ }^{(29)}$. forations results not only in subjective improvement of nasal breathing and nasal symptoms, but also in restauration of the air-conditioning capacity of the nasal airways.

\section{FUNCTIONAL SINUS SURGERY}

Generally, the main goal of functional endoscopic sinus surgery (FESS) is to improve ventilation and drainage of the paranasal sinuses while the mucosal lining is preserved. The crucial region for this surgical procedure is the middle meatus with the opening of the sinuses. Thus, it is highly recommended that the middle turbinate must be protected ${ }^{(30)}$. The influence of the middle turbinate, in relation to the conditioning capacity of the nose, gives rise to controversial discussion ${ }^{(31)}$. In a computer simulation, removal of the middle turbinate resulted in a reduction of $12 \%$ of the nasal air-conditioning capacity compared to healthy nasal passageways ${ }^{(7)}$. So far, the impact of sinus surgery on nasal warming and humidification has not been sufficiently investigated ${ }^{(32)}$. In a study conducted by Keck et al., 22 patients with chronic rhinosinusitis had been examined before and 6-8 weeks after FESS, and results were compared to 22 controls ${ }^{(33)}$. Temperature was measured in the nasal vestibule, the nasal valve region, the anterior turbinate area, and in the nasopharynx. No differences between the patient and the control group concerning temperature profiles were measured. The minimal effect of chronic rhinosinusitis without nasal polyps on the conditioning function of the nose is evident. According to these results, FESS has no major influence on the air-conditioning function of the nose, at least in patients without obstructing nasal polyps.

\section{RADICAL SINUS SURGERY}

The impact of radical sinus surgery without preservation of the mucosal lining on nasal air-conditioning has been investigated by the same research group. Patients with unilateral inverted papilloma were examined ${ }^{(34)}$. Radical maxillary sinus surgery, sphenoidotomy, and ethmoidectomy with complete resection of the lateral nasal wall and the inferior and middle turbinates were performed in all patients. The healthy side side served as control. The temperature and humidity levels in the nasopharynx of the operated and contralateral side were measured. A significantly lower temperature and absolute humidity were registered for the operated side in comparison to non-operated side. These results correspond with those of Drettner et al., who examined four patients with partial maxillectomy ${ }^{(3)}$. They registered significantly reduced nasal humidity and temperature in comparison to normal subjects.

The nasal airflow after radical sinus surgery was also illustrated in computer simulation. The airflow model was based on a CT scan of a patient after radical maxillary sinus surgery and ethmoidectomy, with complete resection of the lateral nasal wall and the turbinates on the right side ${ }^{(35)}$. The calculated nasal volume on the operated side was $145 \mathrm{ml}$, on the healthy side it was $68 \mathrm{ml}$, respectively. The relation between surface and volume amounted to 0.8 for the operated side and 0.3 for the con- 


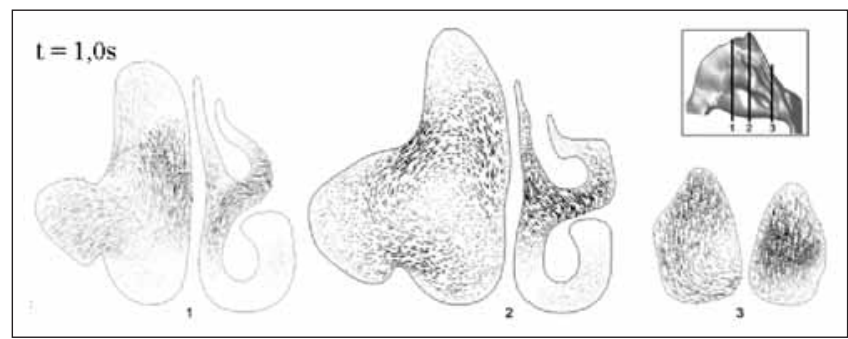

Figure 4. Numerical simulation of inspiratory airflow velocity at three different locations within the nasal airways after unilateral radical sinus surgery ${ }^{(35)}$.

tralateral side. The results of numerical simulation of airflow pattern are shown in Figure 4. The simulation study in the healthy nasal passageways demonstrated only minimal air turbulences towards the nasopharynx. On the operated side, the far more turbulent airflow rebounds directly against the posterior wall of the nose, thus leading to.

\section{CONCLUSION}

Nasal and paranasal surgical interventions have been shown to be associated with improved nasal warming and moistening when the mucosal lining of the nasal passageways is preserved. Even repairs of septal perforations have been demonstrated to elevate intranasal temperature and humidity. Radical sinus surgery with reduction of turbinate tissue leads to reduced nasal heating and humidification within the nasal airways, with an increased risk of nasal dryness and crust formation on the nasal mucosal surface. However, other factors such as the nasal cycle, the airflow distribution in the nasal passageways, and nasal resistance and their impact on nasal climatization have only partially been investigated. Nevertheless, excessive enlargement of the nasal cavity as a result of nasal and sinus operations, especially in the turbinate area, have to be avoided.

\section{REFERENCES}

1. Courtiss EH, Gargan TJ, Courtiss GB. Nasal physiology. Ann Plast Surg 1984; 13: 214-223.

2. Hens G, Hellings PW. The nose: gatekeeper and trigger of bronchial disease. Rhinology 2006; 44: 179-187.

3. Drettner B, Falck B, Simon H. Measurements of the air conditioning capacity of the nose during normal and pathological conditions and pharmacological influence. Acta Otolaryngol 1977; 84: 266277.

4. Svensson S, Olin AC, Hellgren J. Increased net water loss by oral compared to nasal expiration in healthy subjects. Rhinology 2006; 44: 74-77.

5. Assanasen P, Baroody FM, Naureckas E, Solway J, Naclerio RM. The nasal passage of subjects with asthma has a decreased ability to warm and humidify inspired air. Am J Respir Crit Care Med 2001; 164: 1640-1646.

6. Naclerio RM, Pinto J, Assanasen P, Baroody FM. Observations on the ability of the nose to warm and humidify inspired air. Rhinology 2007; 45: 102-111.

7. Naftali S, Rosenfeld M, Wolf M, Elad D. The air-conditioning capacity of the human nose. Ann Biomed Eng 2005; 33: 545-553.

8. Keck T, Leiacker R, Heinrich A, Kuhnemann S, Rettinger G. Humidity and temperature profile in the nasal cavity. Rhinology 2000; 38: 167-171.
9. Keck T, Leiacker R, Riechelmann H, Rettinger G. Temperature profile in the nasal cavity. Laryngoscope 2000; 110: 651-654.

10. Hol MK, Huizing EH. Treatment of inferior turbinate pathology: a review and critical evaluation of the different techniques. Rhinology 2000; 38: 157-166.

11. Jackson LE, Koch RJ. Controversies in the management of inferior turbinate hypertrophy: a comprehensive review. Plast Reconstr Surg 1999; 103: 300-312.

12. Janda P, Sroka R, Baumgartner R, Grevers G, Leunig A. Laser treatment of hyperplastic inferior nasal turbinates: a review. Lasers Surg Med 2001; 28: 404-413.

13. Clement WA, White PS. Trends in turbinate surgery literature: a 35-year review. Clin Otolaryngol Allied Sci 2001; 26: 124-128.

14. Neumann A, Lehmann N, Stange T, et al. Patients' satisfaction after nasal septal and turbinate surgery. Results of a questionnaire. Laryngorhinootologie 2007; 86: 706-713.

15. Corey JP. Acoustic rhinometry: should we be using it? Curr Opin Otolaryngol Head Neck Surg 2006; 14: 29-34.

16. Lindemann J, Keck T, Wiesmiller KM, Rettinger G, Brambs HJ, Pless D. Numerical simulation of intranasal air flow and temperature after resection of the turbinates. Rhinology 2005; 43: 24-28.

17. Moe R. The effect on the respiratory functions of the nose of the lumen-dilatring nasal operations. Acta Otolaryngol (Suppl.) 1942; 45: 5-146.

18. Arunachalam PS, Kitcher E, Gray J, Wilson JA. Nasal septal surgery: evaluation of symptomatic and general health outcomes. Clin Otolaryngol Allied Sci 2001; 26: 367-370.

19. Stewart MG, Smith TL, Weaver EM, et al. Outcomes after nasal septoplasty: results from the Nasal Obstruction Septoplasty Effectiveness (NOSE) study. Otolaryngol Head Neck Surg 2004; 130: 283-290.

20. Bohlin L, Dahlqvist A. Nasal airway resistance and complications following functional septoplasty: a ten-year follow-up study. Rhinology 1994; 32: 195-197.

21. Kemker B, Liu X, Gungor A, Moinuddin R, Corey JP. Effect of nasal surgery on the nasal cavity as determined by acoustic rhinometry. Otolaryngol Head Neck Surg 1999; 121: 567-571.

22. Wiesmiller K, Keck T, Rettinger G, Leiacker R, Dzida R, Lindemann J. Nasal air conditioning in patients before and after septoplasty with bilateral turbinoplasty. Laryngoscope 2006; 116: 890-894.

23. Constantinides MS, Adamson PA, Cole P. The long-term effects of open cosmetic septorhinoplasty on nasal air flow. Arch Otolaryngol Head Neck Surg 1996; 122 :41-45.

24. Rozsasi A, Leiacker R, Kuhnemann S, et al. The impact of septorhinoplasty and anterior turbinoplasty on nasal conditioning. Am J Rhinol 2007; 21:302-306.

25. Fairbanks DN, Fairbanks GR. Nasal septal perforation: prevention and management. Ann Plast Surg 1980; 5: 452-459.

26. Clement PA, Gordts F. Consensus report on acoustic rhinometry and rhinomanometry. Rhinology 2005; 43: 169-179.

27. Lindemann J, Kuhnemann S, Stehmer V, Leiacker R, Rettinger G, Keck T. Temperature and humidity profile of the anterior nasal airways of patients with nasal septal perforation. Rhinology 2001; 39: $202-206$.

28. Pless D, Keck T, Wiesmiller KM, Lamche R, Aschoff AJ, Lindemann J. Numerical simulation of airflow patterns and air temperature distribution during inspiration in a nose model with septal perforation. Am J Rhinol 2004; 18: 357-362.

29. Lindemann J, Leiacker R, Stehmer V, Rettinger G, Keck T. Intranasal temperature and humidity profile in patients with nasal septal perforation before and after surgical closure. Clin Otolaryngol Allied Sci 2001; 26: 433-437.

30. Stammberger H, Posawetz W. Functional endoscopic sinus surgery. Concept, indications and results of the Messerklinger technique. Eur Arch Otorhinolaryngol 1990; 247: 63-76.

31. Kennedy DW, Senior BA. Endoscopic sinus surgery: A review. Prim Care 1998; 25: 703-720.

32. Musebeck K, Rosenberg H. Measurement of temperature in the sinus maxillaris. Laryngol Rhinol Otol 1980; 59: 34-39. 
33. Keck T, Leiacker R, Kuhnemann S, Rettinger G. Heating of air in the nasal airways in patients with chronic sinus disease before and after sinus surgery. Clin Otolaryngol Allied Sci 2001; 26: 53-58.

34. Lindemann J, Leiacker R, Sikora T, Rettinger G, Keck T. Impact of unilateral sinus surgery with resection of the turbinates by means of midfacial degloving on nasal air conditioning. Laryngoscope 2002; 112: 2062-2066.

35. Lindemann J, Brambs HJ, Keck T, Wiesmiller KM, Rettinger G, Pless D. Numerical simulation of intranasal airflow after radical sinus surgery. Am J Otolaryngol 2005; 26: 175-180.
Tilman Keck, MD, Professor and Head Department of Otorhinolaryngology Head, Neck, and Facial Plastic Surgery Elisabethinen Hospital $\mathrm{GmbH}$

Academic Hospital of the Medical University of Graz Elisabethinergasse 14

8020 Graz

Austria

Tel: +43-316-7063-1140

Fax: +43-316-7063-1620

E-mail: tilman.keck@elisabethinen.at 